\title{
Evidence for the Appropriate Use of Telemedicine in Female Pelvic Medicine and Reconstructive Surgery
}

\author{
Francesca Barrett ${ }^{1}$. Lauren E. Stewart ${ }^{1}$ - Benjamin M. Brucker ${ }^{1}$
}

Accepted: 25 October 2021 / Published online: 3 November 2021

(c) The Author(s), under exclusive licence to Springer Science+Business Media, LLC, part of Springer Nature 2021

\begin{abstract}
Purpose of Review To summarize the available literature regarding telehealth interventions in the management of pelvic floor disorders.

Recent Findings Most Female Pelvic Medicine and Reconstructive Surgery (FPMRS) patients own and feel comfortable operating the technology required to participate in telehealth interventions and would be willing to interact remotely with their providers. Telehealth may be an appropriate and effective tool for patient education about bladder and pelvic physiology and pathophysiology, remote pelvic floor muscle strengthening when in-person physical therapy is not accessible, overactive bladder follow-up and medication management, and for postoperative care following uncomplicated incontinence and prolapse surgery.

Summary There is a growing body of literature specific to FPMRS supporting various telehealth interventions that could reasonably be expected to improve access to sub-specialty care while maintaining or improving healthcare quality and reducing costs to the patient and the healthcare system.
\end{abstract}

Keywords Telemedicine in FPMRS $\cdot$ Remote healthcare $\cdot$ Telehealth $\cdot$ Urinary incontinence $\cdot$ Pelvic organ prolapse $\cdot$ Postoperative telehealth

\section{Introduction}

The Center for Medicare and Medicaid Services (CMS) estimates that by 2028 healthcare spending in the USA will reach $20 \%$ of the gross domestic product, far more than any other developed country. [1] Despite this exceedingly high cost of care, the USA consistently lags behind

This article is part of the Topical collection on Reconstructed Bladder Function \& Dysfunction

Benjamin M. Brucker

Benjamin.Brucker@NYULangone.org

Francesca Barrett

Francesca.Barrett@NYULangone.org

Lauren E. Stewart

Lauren.Stewart@NYULangone.org

1 Departments of Urology and Obstetrics and Gynecology, Division of Female Pelvic Medicine and Reconstructive Surgery, NYU Langone Health/NYU Grossman School of Medicine, New York, NY, USA other developed countries with respect to multiple metrics of healthcare quality including chronic disease burden and average life expectancy. [2] Additionally, many Americans lack access to adequate healthcare due to limitations of insurance coverage, local physician shortages, or one of many social determinants of health including race, education, or income. The COVID-19 pandemic has only magnified the issue of healthcare access with an estimated $41 \%$ of US adults delaying or avoiding medical care as of June, 2020. [3]

In an attempt to lower healthcare costs while improving quality and access, many have turned to telemedicine interventions which allow healthcare professionals to evaluate, diagnose, and treat patients in remote locations using telecommunications technology. Increasing data suggests that telemedicine is a cost-effective way to increase efficiency and improve patient satisfaction without sacrificing the quality of care. Applications include monitoring of chronic conditions, such as diabetes, hypertension, and heart failure, detecting early signs of deterioration, providing access to specialty care, such as dermatology and psychiatry, 
Table 1 Terms relevant to the delivery of telehealth services

\begin{tabular}{|c|c|c|}
\hline Term & Definition & Example(s) \\
\hline Telehealth & $\begin{array}{l}\text { An umbrella term to describe any healthcare process occurring virtually } \\
\text { without face-to-face contact }\end{array}$ & $\begin{array}{l}\text { Clinical care and nonclinical services like CME, } \\
\text { meetings, and provider training }\end{array}$ \\
\hline Telemedicine & Virtual technology used specifically between a patient and physician & Clinical care \\
\hline Synchronous & Delivery of health information in real-time & Interactive video conferencing or telephone visit \\
\hline Asynchronous & $\begin{array}{l}\text { The electronic transmission of medical images, records, or pre-recorded } \\
\text { videos through secure email for evaluation at a later time }\end{array}$ & \\
\hline Remote monitoring & The ongoing collection and transmission of health data & Cardiac monitoring, diabetic glucose monitoring \\
\hline MHealth & $\begin{array}{l}\text { The use of a mobile device as the avenue to connect with medical care } \\
\text { through the above methods }\end{array}$ & Smartphone apps \\
\hline
\end{tabular}

delivering patient education for self-management through nursing care, and assessing real-time clinical status such as postoperative assessments [4]. Terminology pertinent to telehealth delivery is reviewed in Table 1.

The COVID-19 pandemic allowed for rapid interest in and expansion of telemedicine, with subsequent changes to telehealth policy and coverage. From a system's perspective, barriers to telemedicine expansion prior to the pandemic included inadequate reimbursement and lack of insurance coverage. In March 2020, CMS expanded telehealth services as a temporary benefit under the Coronavirus Aid, Relief and Economic Security (CARES) Act, which allowed patients to receive insurance coverage for a wider range of medical services. Changes included loosening prior geographic restrictions on telehealth access, alleviating the need for a prior relationship between the provider and patient, allowing for nurse practitioners and physician's assistants to deliver telehealth services, and reimbursing based on medical decision making. In August 2020, federal funding increased for telehealth services, with CMS proposing permanent changes to telehealth benefits. Simultaneous to CMS changes, Health and Human Services (HHS) waived enforcement for HIPAA Rules regarding regulatory requirements for remote communication technologies. [5] The Drug Enforcement Administration loosened requirements on e-prescribing of controlled substances during this time. Finally, many state governments focused on expanding telehealth through Medicaid programs, changing the reimbursement models, and expanding the types of clinicians, facilities, and services eligible for billing. Such efforts allowed for $20 \%$ of medicine to be delivered via telemedicine in 2020. [6]

\section{Telemedicine Adoption and Acceptability in FPMRS}

Interest in expanding telemedicine access within Female Pelvic Medicine and Reconstructive Surgery (FPMRS) increased similarly during the COVID-19 pandemic. This was likely in part because telehealth is well suited to the unique needs of the typical older patient population who are at a higher risk of developing severe COVID-19 symptoms and who often rely on caregivers who were no longer allowed to accompany them during appointments due to limited visitor policies. Additionally, in-person healthcare was heavily restricted for elective or non-urgent conditions during the height of the pandemic, prompting specialists in FPMRS to explore telemedicine as an alternative to ensure patients did not experience long interruptions in their care which could result in negative impacts on the quality of life.

In this environment, academic societies within the field of FPMRS sought to support the utilization of telemedicine. In April 2020, the International Urogynecologic Association (IUGA) provided a framework for the management of pelvic floor disorders during the pandemic using telephone and video visits.[7•] The International Continence Society (ICS) suggested regular follow-up via telemedicine to prevent hospital admissions in neuro-urologic patients.[8•] The American Urogynecologic Society (AUGS) created seminars to address coding, implementation, and integration of telemedicine into routine FPMRS practices, and the Society of Gynecologic Surgeons (SGS) published guidelines for situations appropriate and inappropriate for caring for

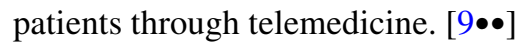

However, the question remained as to whether women with pelvic floor disorders were willing to adopt non-traditional communication methods with their physicians. A timely cross-sectional survey published in March 2020 evaluated the willingness of women with pelvic floor disorders to use mobile technology to communicate with their providers. Two hundred primarily Caucasian patients with a variety of pelvic floor disorders were recruited from an FPMRS clinic at a tertiary center. Women were stratified into younger ( $<45$ years), middle-aged ( $45-65$ years), and older ( $>65$ years) groups. Ninety-two percent owned smartphones, with older women significantly less likely to own a smartphone $(75 \%)$ than middle-aged women $(98 \%)$ and younger women $(100 \%)(p<0.01)$. The majority were capable of using smartphones, with $71 \%$ able to use videoconferencing, $93 \%$ able to send text messages, and $86 \%$ able 
to take pictures or videos. Older women were significantly less likely than young or middle-aged women to express willingness to communicate remotely with their healthcare providers (video-conference, $p=0.02$; internet-based patient portion, $p=0.01$; email, $p=0.001$; text message, $p>0.001$ ). Even so, greater than $66 \%$ of middle-aged women and $50 \%$ of older women were willing to adopt such technologies for communication. Apart from age, educational status also was significantly associated with willingness to use mobile technology. Overall, the authors found that this population did own mobile technology and was able to use the technology, concluding that telemedicine is feasible [10]

\section{Specific Telehealth Applications in FPMRS}

\section{Pelvic Floor Muscle Exercises}

Pelvic floor muscle strengthening is a mainstay of conservative treatment for most pelvic floor disorders. Traditionally, pelvic floor muscle exercises have been performed at home with written instructions from a provider or with the handson guidance of a trained pelvic floor physical therapist. Several studies, which are reviewed below, have evaluated the efficacy of telehealth interventions for pelvic floor muscle strengthening and their impact on urinary incontinence symptom scores.

Smartphone technology, through the use of downloadable applications (apps), can be used to improve patient education, track utilization of resources, and promote adherence with treatment plans through the use of reminders and alarms. Two studies evaluated the use of a pelvic floor muscle training mobile app, Tat, on stress urinary incontinence. A prospective randomized trial of 123 women with SUI ( $>1$ episode/week) evaluated the impact of an app-based treatment program focused on pelvic floor muscle training and lifestyle modification. The control group was a postponed treatment group that did not receive the intervention for 3 months. The primary outcome was SUI symptom severity (ICIQ-UI SF) and condition-specific QoL (ICIQLUTSqol). At 3 months, the intervention group reported improved symptom severity (mean difference - 3.2 (95\% CI -4.3 to -2.1$), p<0.001)$ and condition-specific improvement in quality of life (mean difference -4.6 (95\% CI -7.8 to -1.4$), p=0,005)$. Secondary outcomes similarly were improved, with a decrease in median leakage episodes/ week $(p=0.001)$, overall improvement in urinary leakage $(p<0.001)$, and reduction in incontinence aid use $(p=0.023)$. [11] A follow-up study evaluated 2-year outcomes from the same trial and was able to retain $75.4 \%$ of the original intervention group $(n=46 / 62)$. At 2 years, there was a sustained improvement in incontinence and quality of life scores, with $63.1 \%$ reporting global symptom improvement (8.7\% very much improved, $19.6 \%$ much better, $34.8 \%$ a little better). During the follow-up period, $21.7 \%$ of women sought alternative treatment for their SUI including $4.1 \%$ who underwent surgery. When those who had surgery were excluded from the analysis, the results were not significantly different. The authors concluded that the Tat application had a long-term clinically relevant impact on SUI. [12]

Another randomized controlled trial evaluated the impact of asynchronous internet-based pelvic floor physical therapy (PFPT) on symptoms of SUI. Two hundred fifty women were randomized to either a 3-month, internet-based treatment group that included tailored email support from a physical therapist, cognitive behavioral therapy, and escalating levels of treatment, or to a postal mail-based program that included PFPT exercises, basic information on SUI, and lifestyle advice. The primary outcome was symptom severity (ICIQ-UI) at 1 year and 2 years. Secondary outcomes included PGI-I, health-specific QoL metrics, and patient satisfaction. There was a high loss to follow-up with $32.4 \%$ and $38 \%$ of participants lost at 1 year and 2 years, respectively. However, the authors found that both groups had significant improvement in symptom and condition quality of life compared with baseline $(p<0.001)$, with no significant difference between groups. After 2 years, a higher proportion of participants in the internet group reported much or very much improvement (39.2\% vs $23.8 \%, p=0.03)$. The authors concluded that even a short course ( 3 months) of non-face-to-face treatment with PFPT provided significant and clinically relevant improvements in SUI at 1 year and 2 years. [13]

Poor adherence to pelvic floor muscle exercises has long been known to limit their long-term efficacy. A small randomized controlled trial aimed to assess whether a mobile application could improve adherence to strengthening exercises compared with traditional paper-based instructions. Twenty-one patients were randomized to the app group consisting of pelvic floor exercises and the visual component of electromyography, or to the control group, which included only written instructions for pelvic floor exercises. Subjects were asked to complete exercises twice daily (mobile app groups would receive reminders) with evaluations at the 1-, 2-, and 3-month time. Adherence to the exercise schedule was higher in the mobile application group at 2 months $(49.8 \pm 8.1$ vs $33.6 \pm 10.7)$ and 3 months $(43.8 \% \pm 8.7$ vs $17.7 \% \pm 6.3 \%$ ), with $p<0.001$. Adherence decreased over time for both groups, though at a higher rate in the control. All secondary outcomes showed improvement over the 3 -month study period without differences between the app and control group ( $p=0.887$ vaginal symptoms, $p=0.573$ QoL, $p=0.825$ SUI). The authors concluded that the mobile app increased adherence to home exercise compared to written instructions but did not change clinical outcomes. [14] 
The above papers have all compared telehealth pelvic exercise interventions to paper-based or traditional controls which may be less effective than formal, in-person, pelvic floor physical therapy. To address this, a small non-inferiority RCT $(n=54)$ randomized women with SUI or SUI predominant-mixed incontinence to a home vaginal biofeedback device with online support or to 12 weeks (4 visits) of in-person, supervised pelvic floor physical therapy. The primary outcome, assessed at 3 months, was the severity of UI and quality of life (ICIQ-SF), and secondary outcomes included sexual function, $\mathrm{OAB}$ symptoms, patient satisfaction, and global impression of improvement. In the intent-totreat analysis, home biofeedback was non-inferior to PFPT, and both groups showed improvement and meaningful clinical effect in UI severity $(p=0.009)$. When analyzing for compliance through per-protocol analysis, non-inferiority was still observed for highly compliant patients, though the effect was lost for moderately compliant patients. Several barriers to adherence were noted for both groups: Cost, distance to care, and need to miss work were barriers to PFPT; difficulty using the device, discomfort, or lack of personal interaction to encourage compliance were barriers to home biofeedback. The authors concluded that home biofeedback for the treatment of SUI was non-inferior to standard care and may be effective when in-person PFPT is not available or feasible. [15]

The past 10 years have seen an enormous rise in the number of mobile apps coming to the market, and those focused on the treatment of pelvic floor disorders have been no exception. One study addressed the potential variability in app functionality, features, and accuracy (i.e., whether the mobile application's recommendations were in accordance with published scientific literature and national guidelines) using a standardized scoring system. They evaluated 32 unique mobile applications (15 paid, 17 free) that were functional, available in English, required no biofeedback device, and were intended for non-pregnant patients. Although paid applications had more privacy features, images, references to primacy literature, and tech support, the overall scores between the two groups were nearly identical (9.93 paid apps vs 9.41 free apps). The study identified the two highest-rated applications, both with a score of 12 (out of 15), as Kegel Trainer (free) and Kegel Trainer Pro (paid). [16]

Overall, telehealth interventions aimed at pelvic floor muscle strengthening had benefits over traditional models (written information and physician teaching) in multiple studies and were non-inferior to supervised pelvic floor physical therapy in one study. These interventions are inexpensive, have very few potential harms, and should be considered an effective first-line alternative for patients with urinary incontinence who lack access to trained pelvic floor physical therapists.

\section{Postoperative Care}

Postoperative care presents a unique opportunity for the incorporation of telemedicine to maintain quality of care while reducing healthcare-related costs. There is no standard recommended schedule for postoperative visits after FPMRS surgery, although many providers suggest two postoperative visits during the typical 90-day global billing period. Utilizing virtual visits for at least one postoperative encounter can help to identify and triage issues requiring additional workup or management while saving those patients who are doing well the time required to make a special trip to the provider's office.

Three studies have evaluated the use of telemedicine for follow-up after midurethral sling (MUS) surgery. Presented at the 2020 American Urological Association meeting but not yet published, a multi-institutional randomized controlled equivalence trial compared telemedicine-based follow-up (TBFU) to office-based follow-up (OBFU) for a 3-week postoperative visit following MUS and enrolled 237 patients. Outcomes included patient satisfaction and unplanned events. The abstract, published in April 2020, reported that TBFU and OBFU patients were equally satisfied $(71.1 \%$ vs. $69 \%, p=0.2)$. There was no difference in hospital admissions, ED visits, unplanned office visits, unplanned phone calls, or adverse medical events. TBFU patients were more compliant with 3-5-month OBFU (90.1\% vs $79.3 \%, p=0.04)$, but $13.2 \%$ of TBFU $(16 / 121)$ requested crossover to OBFU, with patients older than 65 having a higher odds of requesting crossover $(p=0.048$, OR:3.3, 95\% 1.1-9.9). [17].

A prospective observational study of 356 patients undergoing MUS over a 5-year period utilized telephone follow-up for 262 patients, of which 90\% (234/262) required no additional in-person follow-up. Ten percent (28/262) required an office visit based on symptoms expressed during the tele visit, ranging from persistent SUI symptoms, pain, recurrent infection, symptoms of tape erosion, voiding difficulty, prolapse, or urinary urgency. The remaining participants (92/356) were triggered for outpatient follow-up at the time of surgical discharge based on surgeon discretion/ preference. Patients in the telephone follow-up group had similar satisfaction scores to the in-person follow-up group, although this study had several limitations, namely its nonrandomized design. [18]

Most clinicians agree that at least one postoperative visit should be in-person, so the optimal timing of telemedicine visits remains a viable question. A prospective cohort study evaluated the feasibility, reliability, and satisfaction with postoperative telephone follow-up 1 year after urogynecologic surgery. The study included 215 women who underwent MUS and 205 who underwent anterior colporrhaphy (with or without permanent mesh). 
The reliability of the telephone assessment was evaluated with an in-person visit 7-12 days later. In the MUS group, patients who were continent had a high concordance correlation (K 1.0) and those who were incontinent had moderate concordance correlation (K 0.7) between remote and in-person diagnoses, likely because patients misinterpreted de-novo urgency incontinence as recurrent SUI. In the prolapse group, the telephone visit was able to detect recurrent prolapse (K 0.97) but could not provide prolapse staging information. In both groups, mesh extrusions were exclusively detected at the in-person clinic visit ( $\mathrm{K} 0.0)$. Satisfaction with telephone follow-up was high in both groups. The authors concluded that telephone follow-up 1 year after surgery is a reliable tool for continent patients following MUS and for patients following prolapse repair without the use of permanent mesh. [19]

In contrast to remote follow-up 1 year after surgery, a non-inferiority RCT evaluated patient satisfaction with a schedule of virtual and in-person visits for the first 3 months of postoperative recovery after prolapse surgery. The telemedicine group received a video call from a nurse 48-72 h after discharge, a video visit 30 days, and an in-person visit 90 days after surgery. The control group had in-office visits 30 days and 90 days after surgery. There was no difference in mean patient satisfaction scores, consistent with non-inferiority ( 80.7 vs 81.2 ). However, more patients in the virtual group were pleased with the quality of the visit, reporting that their visit was on time and efficient $(P<0.001)$, whereas more women in the office group felt as if their visit took longer than expected $(P<0.001)$. Postoperative complication rates were $31 \%$ in the virtual group and $46 \%$ in the office group $(P=0.3)$, with the most common intervention being the administration of antibiotics. No difference was noted in unscheduled phone calls, office visits, ED visits, or readmissions within 90 days of surgery. The authors concluded that patient satisfaction with televisits was non-inferior to traditional office postoperative visits and were reasonable alternatives for women undergoing prolapse surgery. [20]

Another non-inferiority RCT evaluating patient satisfaction, safety, and clinical outcomes of televisit visits versus in-person postoperative visits following FMPRS surgery. One hundred women who were scheduled for any outpatient or inpatient pelvic surgery were randomized to 2-, 6-, and 12-week postoperative visits either by RN telephone or by clinic visit. The authors found that global assessment of surgeon rating by patients in the telephone group was non-inferior to the clinic group ( $92 \%$ clinic vs $88 \%$ ). Notably, surgeon communication was rated as inferior in the televisit group, likely as the patient communicated primarily with a nurse. There were no differences in adverse events $(p=0.36)$, with UTI accounting for $58 \%$ of total adverse events. Rates of readmission, ED, and PCP visits were equivalent. Symptom scores (PFDI-20) improved in both groups postoperatively without differences between groups (all outcome metrics, $p>0.05$ ). The authors concluded that telephone follow-up after the broad spectrum of pelvic floor surgery was non-inferior in terms of satisfaction, clinical outcomes, or adverse events. [21]

Ultimately, studies indicate that incorporating telemedicine into postoperative care for FPMRS patients is safe and generally results in similar patient satisfaction scores. The degree to which postoperative care is converted to virtual visits remains an area of study and may have implications for the diagnosis of delayed complications and patient perception of surgeon communication.

\section{Urgency Urinary Incontinence}

Urgency urinary incontinence (UUI) and overactive bladder $(\mathrm{OAB})$ are particularly amenable to telehealth interventions since, aside from the initial assessment and third-line therapy, much of the management relies heavily on patient education, lifestyle modification, and medication management which lend themselves well to remote healthcare delivery. UUI/OAB have been the focus of several telehealth-based studies.

An RCT compared a video-based continence program to usual care in 64 older women (mean 73 years) with urinary incontinence. All patients received an initial in-person biofeedback-assisted pelvic floor muscle training session, followed by telemedicine or in-person behavioral management program. The authors found that both groups experienced fewer daily incontinence episodes $(p<0.001)$, fewer daily voids $(p<0.001)$, higher volume of urine at each void $(p<0.005)$, and improvement in pelvic floor muscle strength $(p<0.005)$ compared with baseline. No difference in outcomes was found between the groups, suggesting that virtual visits are a good alternative to in-person health education in an older population. [22]

While a history, physical exam, and urinalysis (UA) are considered the minimum workup for patients presenting with $\mathrm{OAB}$, for most patients, the first consultation visit consists disproportionately of counseling. Researchers in the UK performed an RCT comparing telephone vs in-person first consultation visits for patients with urinary incontinence who had a prior documented UA and physical exam available in their records. One hundred ninety-five patients were randomized. The primary outcome was a short-term clinical outcomes scale (PEQ) assessed at 6 months. The authors found no difference in quality outcomes, while telemedicine visits were shorter (10.94 min vs $25.9 \mathrm{~min}$ ), had lower personal costs, and had patient-reported-enhanced communication. The study did note that standard care was overall more cost-effective, largely driven by an increased number of in-office follow-up visits during the study period in the intervention group. [23] 
Subsequently, a small, single-center, prospective RCT was presented, evaluating whether telemedicine follow-up utilizing a guideline-driven care pathway could improve patient-reported outcomes in OAB. The researchers recruited 23 patients with isolated, idiopathic OAB not previously treated with $>1 \mathrm{OAB}$ medication or third-line treatments. A telephone call between office visits, with both groups, followed for at least 12 weeks after the initial visit, was compared with usual care. More patients in the telemedicine group progressed to third-line treatment and were receiving treatment at the end of the study. Both groups had a reduction in symptom bother and improved quality of life (OABq-SF). Despite their small sample size, the authors concluded that telemedicine follow-up may improve medication compliance and progression to third-line agents in OAB. [24]

While the published data regarding telehealth specifically for $\mathrm{OAB}$ is not as robust as other conditions in FPMRS, the typical treatment pathway lends itself well to telehealth interventions. The available data suggests that care may be more cost-effective when the initial consultation is in-person, but follow-up for first- and second-line therapy is likely safe and may improve adherence to care and progression to third-line treatment.

\section{Other Applications}

Telemedicine interventions may be an appropriate component of care for several other conditions within FPMRS. These conditions include interstitial cystitis/bladder pain syndrome (IC/BPS), pessary management, and recurrent urinary tract infection (UTI).

There is limited data regarding remote healthcare delivery in IC/BPS. However, given that the first stages of treatment are characterized by patient education, counseling regarding lifestyle modification, and medication management, IC/BPS may be another appropriate condition for the integration of telehealth interventions. A small RCT enrolled 60 patients to compare the effectiveness of video-based m-health educational tool with standard care. The m-health intervention included weekly educational videos on health education and symptom self-management. It should be noted that the $m$-health intervention was in addition to, rather than instead of, usual care. Patients who received m-health support exhibited significant improvement in disease severity and quality of life compared with the control group. [25]

Regarding pessary management and recurrent UTI's, an excellent literature review provided generalized guidance on incorporating virtual management into FPMRS practice during the COVID-19 pandemic. While there are no studies specifically addressing telehealth interventions for pessary management, there are several studies reporting outcomes with continuous pessary use (no interval cleaning or exam) from 6 to 24 months. These studies suggest that risks of pessary-related adverse events (erosion or bleeding $5.0 \%$ (95\% CI 1.9, 9.0); discharge 5.8\% (95\% CI 3.6, 8.5); vaginitis $1.8 \%$ (95\% CI 0.2, 4.6); voiding dysfunction $4.7 \%(95 \%$ CI $1.4,9.8)$; fistula $0 \%(95 \%$ CI $0,1.1))$ are overall low, suggesting that patients can safely extend the time between pessary cleanings to 6 months and up to 24 months with minimal adverse events. Although it has not been studied, it is reasonable to conclude that, if spacing in-person pessary maintenance visits to 6-24 months, interval telemedicine follow-up could be utilized to review symptoms and prompt an earlier in-person visit if new or unusual symptoms have

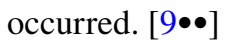

The above review also discussed how telemedicine-based assessment and treatment of recurrent UTIs can be incorporated into practice. However, it should be noted that their recommendations were specific to the unique circumstances of the COVID-19 pandemic. Empiric treatment of uncomplicated cystitis in women is well-established, safe, and effective, but the empiric treatment of recurrent UTIs carries added risks including increased/unnecessary prescribing and antibiotic resistance, so it should be avoided when possible. The safety and efficacy of telehealth interventions involving empiric antibiotic treatment of recurrent UTIs outside of the COVID-19 pandemic have not been studied. A reasonable way to incorporate telehealth into the management of recurrent UTIs is with a telemedicine-based symptom assessment with urine testing, if indicated, performed at the patient's local medical laboratory and used to guide therapy.

\section{Conclusions}

There is a growing body of literature supporting various telehealth interventions in the field of Female Pelvic Medicine and Reconstructive Surgery that could reasonably be expected to improve access to sub-specialty care while maintaining healthcare quality and reducing costs to the patient and the healthcare system.

Studies have already suggested that smartphone app and internet-based interventions may improve compliance with pelvic floor muscle strengthening programs compared with traditional written or verbal instruction and this increase in compliance may result in improved outcomes for patients with urinary incontinence. The same telehealth-based interventions are a reasonable alternative to supervised PFPT if the latter is not accessible. Telemedicine evaluations can safely be incorporated into the postoperative care of patients following MUS and native tissue prolapse repair without increasing adverse events. The initial evaluation of $\mathrm{OAB} / \mathrm{UUI}$ may be more cost-effective when performed in person, but subsequent telehealth follow-up may improve adherence to medication management and increase rates of 
progression to third-line therapy. Several other disease states commonly treated by FPMRS specialists (IC/BPS, pessary management, and recurrent UTI) may lend themselves well to telehealth interventions; however, data supporting safety, efficacy, and acceptability remains sparse.

While most of the studies reviewed above are randomized controlled trials, many are limited by small sample sizes and relatively short duration of follow-up. More robust research is needed to help inform guidelines for the appropriate use of telehealth interventions as we begin to re-shape our practices, incorporating lessons learned from the COVID-19 pandemic.

\section{Declarations}

Conflict of Interest Francesca Barrett and Lauren Stewart declare that they have no conflicts of interest. Benjamin Brucker is a speaker and consultant for Urovant and Allergan, an investigator for Boston Scientific, and an advisor for Watkins-Conti and Click Therapeutics.

\section{Bibliography}

Papers of particular interest, published recently, have been highlighted as:

- Of importance

$\bullet$ Of major importance

1. Keehan SP, Cuckler GA, Poisal JA, et al. National health expenditure projections, 2019-28: expected Rebound in prices drives rising spending growth. Health Aff (Millwood). 2020;39:70414. https://doi.org/10.1377/hlthaff.2020.00094.

2. MK TRA. U.S. Health care from a global perspective, 2019: higher spending, worse outcomes? The Commonwealth Fund 2020 January 30, 2020.

3. Czeisler ME, Marynak K, Clarke KEN, et al. Delay or avoidance of medical care because of COVID-19-related concerns United States. MMWR Morb Mortal Wkly Rep. 2020;69:1250-7. https://doi.org/10.15585/mmwr.mm6936a4.

4. Flodgren G, Rachas A, Farmer AJ, et al. Interactive telemedicine: effects on professional practice and health care outcomes. Cochrane Database Syst Rev 2015: CD002098. https://doi.org/ 10.1002/14651858.CD002098.pub2.

5. (HHS) UDoHaHS. Notification of enforcement discretion for telehealth remote communications during the COVID-19 nationwide public health emergency. 2021.

6. Doximity. 2020 State of Telemedicine report: examining patient perspectives and physician adoption of telemedicine since the COVID-19 pandemic. 2020.

7. Ranee Thakar DR, Angie Rantell, Wendy Ness, Maura Seleme, Bary Berghmans. Guidance for the management of urogynecological conditions during the coronavirus (COVID-19) pandemic. 2020. (Guidance from the International Urogunecologic Association (IUGA) on the safe management of pelvic floor conditions during the COVID pandemic.)

8. Huri E, Hamid R. Technology-based management of neurourology patients in the COVID-19 pandemic: is this the future? A report from the International Continence Society ICS institute.
Neurourol Urodyn. 2020;39:1885-8. https://doi.org/10.1002/ nau.24429 ((Guidance from the International Continence Society on telehealth management of patients with neurologic conditions impacting bladder function.)).

9. Grimes CL, Balk EM, Crisp CC, et al. A guide for urogynecologic patient care utilizing telemedicine during the COVID19 pandemic: review of existing evidence. Int Urogynecol J. 2020;31:1063-89. https://doi.org/10.1007/s00192-020-043144 (This is an outstanding review published at the height of the COVID-19 pandemic which includes a comprehensive review of available literature as well as expert guidance for clinical scenarios in which it is appropriate and inappropriate to incorporate telehealth services into FPMRS practice).

10. Lee DD, Arya LA, Andy UU, et al. Willingness of women with pelvic floor disorders to use mobile technology to communicate with their health care providers. Female Pelvic Med Reconstr Surg. 2019;25:134-8. https://doi.org/10.1097/SPV.0000000000 000668.

11 Asklund I, Nystrom E, Sjostrom M, et al. Mobile app for treatment of stress urinary incontinence: a randomized controlled trial. Neurourol Urodyn. 2017;36:1369-76. https://doi.org/10. 1002/nau.23116.

12 Hoffman V, Soderstrom L, Samuelsson E. Self-management of stress urinary incontinence via a mobile app: two-year follow-up of a randomized controlled trial. Acta Obstet Gynecol Scand. 2017;96:1180-7. https://doi.org/10.1111/aogs.13192.

13. Sjostrom M, Umefjord G, Stenlund $\mathrm{H}$, et al. Internet-based treatment of stress urinary incontinence: 1- and 2-year results of a randomized controlled trial with a focus on pelvic floor muscle training. BJU Int. 2015;116:955-64. https://doi.org/10.1111/bju. 13091.

14 Araujo CC, Marques AA, Juliato CRT. The adherence of home pelvic floor muscles training using a mobile device application for women with urinary incontinence: a randomized controlled trial. Female Pelvic Med Reconstr Surg. 2020;26:697-703. https://doi.org/10.1097/SPV.0000000000000670.

15. Barnes KL, Cichowski S, Komesu YM, et al. Home biofeedback versus physical therapy for stress urinary incontinence: a randomized trial. Female Pelvic Med Reconstr Surg $2020 \mathrm{https}: / /$ doi. org/10.1097/SPV.0000000000000993.

16. Barnes KL, Dunivan G, Jaramillo-Huff A, et al. Evaluation of smartphone pelvic floor exercise applications using standardized scoring system. Female Pelvic Med Reconstr Surg. 2019;25:328-35. https://doi.org/10.1097/SPV.0000000000 000563 .

17. Derisavifard SRJ, Kocher N, Giusto L, Zahner P, Luo D, Dielubanza E, Li J, De Jesus MR, Gomelsky A. BalzarroM, Rackley R, VasavadaS, Moore C, Goldman H. Telemedicine optimizes early postoperative follow up after synthetic mid-urethral sling (MUS): a randomized, multi-institutional control trial [MP3017]. J Urol. 2020;203:114-5.

18. Jefferis H, Muriithi F, White B, et al. Telephone follow-up after day case tension-free vaginal tape insertion. Int Urogynecol J. 2016;27:787-90. https://doi.org/10.1007/s00192-015-2891-6.

19 Balzarro M, Rubilotta E, Trabacchin N, et al. A prospective comparative study of the feasibility and reliability of telephone follow-up in female urology: the patient home office novel evaluation (PHONE) study. Urology. 2020;136:82-7. https://doi.org/ 10.1016/j.urology.2019.10.021.

20. Lee DD, Arya LA, Andy UU, et al. Video virtual clinical encounters versus office visits for postoperative care after pelvic organ prolapse surgery: a randomized clinical trial. Female Pelvic Med Reconstr Surg. 2020. https://doi.org/10.1097/SPV. 0000000000000909. 
21 Thompson JC, Cichowski SB, Rogers RG, et al. Outpatient visits versus telephone interviews for postoperative care: a randomized controlled trial. Int Urogynecol J. 2019;30:1639-46. https://doi. org/10.1007/s00192-019-03895-z.

22 Hui E, Lee PS, Woo J. Management of urinary incontinence in older women using videoconferencing versus conventional management: a randomized controlled trial. J Telemed Telecare. 2006;12:343-7. https://doi.org/10.1258/135763306778682413.

23 Jones G, Brennan V, Jacques R, et al. Evaluating the impact of a 'virtual clinic' on patient experience, personal and provider costs of care in urinary incontinence: a randomised controlled trial. PLoS One. 2018;13:e0189174.

24. Palmerola REC, Sussman R, Brandon C, Smilen S, Pape D, Rosenblum N, Brucker B. Can telemedicine improve follow up adherence and outcomes in patients with overactive bladder? Neurourol Urodyn. 2020;39:S108.

25. Lee MH, Wu HC, Tseng CM, et al. Health education and symptom flare management using a video-based $m$-health system for caring women with IC/BPS. Urology. 2018;119:62-9. https:// doi.org/10.1016/j.urology.2018.05.027.

Publisher's note Springer Nature remains neutral with regard to jurisdictional claims in published maps and institutional affiliations. 